\title{
TRANSFORMACIONES HISTÓRICAS EN LA MANERA DE OPERAR DE LAS FUERZAS MILITARES: REFLEXIONES A partir de la Operación Colombia
}

\author{
General (RA) FLAVIO ULLOA ECHEVERRY ${ }^{1}$
}

\section{INTRODUCCIÓN}

Las Fuerzas Militares en una democracia tienen como objetivo garantizar todos los esfuerzos para debilitar o destruir las amenazas bélicas que operan por fuera de los límites territoriales, proveer la seguridad interna contra la amenaza subversiva de fuerzas internas que pretendan debilitar o destruir el Estado y sus instituciones dentro de su territorio, y también aportar a la seguridad interna apoyando el desarrollo de la Nación o cuando aparecen amenazas que reducen el poder del Estado. Para que las Fuerzas Militares puedan operar requieren de una política institucional que le provea los lineamientos y los requerimientos para su actuación en términos de la voluntad política y el soporte del Estado. De la política institucional se deriva la política operacional, que determina como ellas deben actuar.

Examinar cómo las Fuerzas Militares de Colombia han venido operando en el último siglo y en especial el desarrollo que han tenido desde el año 2000 al 2015 es el propósito de este trabajo, además de hacer un análisis en los aspectos que mejoraron ostensiblemente su actuación para lograr una victoria sobre la subversión, el terrorismo, el narcotráfico y las organizaciones criminales. Hay que incluir en este trabajo a la Policía Nacional, que en Colombia ha jugado un papel importante, pero que además a partir de la segunda mitad del siglo XX se reestructuró en una institución dependiente del Ministerio de Defensa Nacional, integrándose así en este ente gubernamental a la defensa y la seguridad de la Nación. Este aspecto es un diferenciador notable con la mayoría de países en el mundo, $\mathrm{y}$ ha venido jugando un papel decisorio en el resultado final.

Aquellos aspectos que permitieron un desarrollo muy importante de la Fuerza Pública colombiana (Fuerzas Militares y Policía Nacional) son los siguientes: 
La cultura militar, la organización, la tecnología, el desarrollo de capacidades especiales, la inteligencia militar y la estrategia de defensa y seguridad. Todos estos aspectos fueron reconocidos en su conjunto por el Pentágono en el marco de los encuentros y conversaciones operacionales entre las dos fuerzas en las cuales participé en el 2013 como jefe de la delegación colombiana; y por la OTAN y las fuerzas de defensa europeas en el marco de la visita de nuestro ministro de Defensa Juan Carlos Pinzón Bueno, en la cual participé como Jefe del Estado Mayor Conjunto. Este análisis estará determinado en el tiempo desde los años sesenta hasta 2015 , no sin antes dejar en claro y en forma muy breve algunos antecedentes históricos.

Si bien el Ejército de Colombia y la Armada tienen sus orígenes con el Ejército Libertador $^{2}$, el Ejército viene a tener una estructura institucional a partir de la reforma militar del presidente Rafael Reyes. "Reyes comprendió la necesidad de contar con unas Fuerzas Armadas permanentes, profesionales, adecuadamente estructuradas y neutrales en materia política. Se inició en 1906, la expedición de una serie de decretos que permitieron la reorganización del régimen salarial y de las zonas militares que existían en el país, así como la contratación de la Misión Militar Chilena, que condujo a la fundación de la Escuela Militar de Cadetes de San Diego". La Armada República de Colombia, tras sucesivos cambios y restructuraciones, toma en 1936 la forma institucional que requería nuestro país.

La Aviación Militar nace en 1919 durante el gobierno del presidente Marco Fidel Suárez, y durante el conflicto con el Perú actúa como una fuerza independiente, bajo el comando del Cr Herbert Boy y con comunicación directa con el presidente Enrique Olaya Herrera. En 1942 alcanzó su plena autonomía y se estructura como la Fuerza Aérea Colombiana.

Durante el Conflicto con el Perú las Fuerzas Militares tienen una destacada actuación que permitió, por una parte, recuperar nuestra integridad territorial y dar un avance significativo a la capacidad militar de nuestra Nación, en especial a la Fuerza Aérea y a la Armada que en muy poco tiempo se equiparon y organizaron para entrar en la guerra, y por otra alcanzar el reconocimiento y la valoración de nuestro territorio.

En las décadas de los años cuarenta y cincuenta Colombia se ve envuelta en una violencia partidista política que afecta notablemente el desarrollo del país, y que también involucra a las Fuerzas Militares. Entre 1951 y 1956 Colombia participa con el Ejército y la Armada en la guerra de Corea, lo que hace que las Fuerzas Militares no solamente sean bien equipadas sino que además reciban capacitación y entrenamiento que mejoraron enormemente sus capacidades. 
Después de este rápido resumen, entro a realizar el análisis sobre el desarrollo militar colombiano. La cultura militar colombiana se analiza verificando algunos aspectos como la disciplina, la moral de las tropas, su educación, las relaciones civiles militares y el desarrollo del derecho de la guerra. En cuanto a la moral de la tropa, se puede definir como "Aquellos principios de orden espiritual -no jurídico- que deben regular la actividad de los miembros de la Institución Militar para el mejor cumplimiento de los fines de esta última que son la defensa militar del país". El general Montgomery da una definición que en últimas nos aclara que es la moral militar: "El factor individual más importante para la obtención del triunfo es el espíritu del combatiente".

Encontramos dos afectaciones graves en la moral militar colombiana, una en los años ochenta durante el gobierno del presidente Belisario Betancur y otra, tal vez la más importante, durante el gobierno de Ernesto Samper, cuando se vivió una de las mayores crisis institucionales desde los años cuarenta, que afectó notablemente a las Fuerzas Militares. Así mismo, constató la presencia de una moral alta en los años sesenta y setenta, y una moral muy alta entre 2001 y 2015, producto de la exigencia y la definición clara de los objetivos de las Fuerzas Militares y un crecimiento institucional en nuestro país.

La disciplina militar va muy emparejada con la moral, que igualmente tiene su mayor afectación en los años noventa, por la falta de definir claramente los roles y misiones de las Fuerzas Militares ante una creciente escalada terrorista y de narcotráfico en medio de la crisis política que se estaba viviendo. Ante el vacío de poder estatal y la falta de voluntad política para definir la política de seguridad y defensa, los particulares y las mafias narcotraficantes crearon grupos armados que complicaron aún más la situación y que terminaron afectando esta moral y disciplina militar. La disciplina tuvo su mejor desempeño entre los años sesenta y setenta, y mejoró notablemente a partir del 2001, con su mejor calificación entre 2012 y 2013.

La educación es una variable muy importante en el desempeño militar y en la configuración de la cultura. Hay dos hitos importantes: uno, la profesionalización del oficial, que se inicia en los años noventa con la implementación de las carreras profesionales liberales dentro de las Escuelas Militares, la educación tecnológica de los suboficiales, la creación en el año 2000 de los soldados profesionales y sus escuelas, y dos, la incorporación de las especializaciones y maestrías en las Escuelas de postgrados y la Escuela Superior de Guerra. La educación ha venido siempre en un proceso de mejoramiento continuo, y en particular a partir de 2006, cuando se dio un especial énfasis y seguimiento por parte del Ministerio de Defensa y del Comando General de las Fuerzas Militares.

Las relaciones civiles-militares, conocidas como la confianza institucional entre el gobierno, el Estado y los civiles con sus Fuerzas Militares, tienen su 
mayor crisis durante el gobierno de Samper, y en segundo término con Betancur, por la falta de confianza de los militares en especial en su Comandante en Jefe, a quien en el primer caso no ven con la autoridad moral requerida para dirigir el país ni las Fuerzas, y en el segundo por su falta de apoyo y respaldo institucional. Encontramos las mejores relaciones en los años sesenta y setenta y posteriormente empiezan a mejorar a partir del 2000 hasta llegar a su mejor época después de 2005.

En cuanto al derecho de la guerra, fue afectado enormemente por los acontecimientos del país en una conjugación de los fenómenos delictivos de terrorismo y narcotráfico y la ausencia de políticas claras de gobierno sobre la seguridad interior en nuestro país. Siempre en las escuelas se educó al oficial y al suboficial y se entrenó al soldado en un marco de cumplimiento legal y normativo. Sin embargo, a partir del año 2001 se enfatiza en la educación y entrenamiento en derechos humanos y el Derecho Internacional Humanitario, se definen las reglas de encuentro donde la Fuerza Aérea es pionera en su aplicación, y hay un desarrollo muy importante en la creación de los oficiales de derecho operacional y los comités jurídicos operacionales que asesoran y apoyan a los comandantes en la toma de decisiones.

\section{La Operación Colombia}

La Operación Colombia, iniciada el 9 de diciembre de 1990, constituye un hecho histórico, que se enmarca en una multiplicidad de interpretaciones y que para algunos fue un acto que cerró de frente el diálogo y la posibilidad de paz con las FARC. Para otros, fue un acto que frenó la capacidad estratégica y las dinámicas de crecimiento de las FARC y el ELN, en el marco de una Coordinadora Guerrillera "Simón Bolívar". Pero en lo que todos coinciden es en que esta acción planteó un antes y un después para la dinámica del conflicto armado en Colombia.

"El 9 de diciembre de 1990 tropas de la Séptima Brigada del Ejército de Colombia, comandadas por el general Luis Humberto Correa, realizaron la primera gran operación aeroterrestre contra un complejo narcoterrorista que constituía la sede del Secretariado de las FARC, conocida con el mítico nombre de Casa Verde, en la zona rural Uribe-Meta sobre las estribaciones surorientales del Páramo del Sumapaz. Al decir de Jacobo Arenas ideólogo fundador del grupo terrorista, "Casa verde, era el símbolo de la construcción de un movimiento revolucionario que orientaba guerrillas comunistas, como un estímulo a la subversión para la toma del poder". Golpear al Secretariado de las FARC en su sede era asunto de máxima trascendencia estratégica para el Ejército Nacional y de singular importancia política para el país. En ese orden de ideas, la Operación Colombia, se planeó en detalle con base en testimonios de desertores de las FARC, aerofotografías de la Fuerza Aérea, análisis meteorológicos y selección de tropas de asalto, cierres sobre el terreno, fuegos aerotácticos y maniobras sobre los objetivos concretos. El asalto aeroterrestre después de los fuegos de ablandamiento aerotácticos, fue conducido por 
las Fuerzas Especiales. Los comandos desembarcaron sobre los objetivos principales:

Casa de Tirofijo, casa de Jacobo, Aulas, Escuela de Cuadros y depósitos logísticos ${ }^{33}$ ".

Esta es una de las descripciones sobre la Operación Colombia que se pueden encontrar tanto en revistas académicas como en la prensa en general, en la que se sigue hablando del tema luego de más de 20 años no solo por la importancia que tuvo en el ámbito nacional de seguridad, sino por la importancia que generó en los temas del pensamiento estratégico operacional. La Operación Colombia ha sido analizada en distintas publicaciones periodísticas y académicas en las que se ha reflexionado sobre su pertinencia y su necesidad en el contexto constituyente que vivía el país en los inicios de la década del noventa.

Una de las múltiples voces frente a este hecho es justamente la de los militares. Recuperar sus vivencias y memorias constituye un aporte para nutrir el pensamiento estratégico operacional, y una reflexión sobre una de las operaciones militares más importantes en la historia del conflicto armado colombiano, como quiera que se inició un proceso de debilitamiento del Bloque Oriental de las FARC.

Para el caso específico de la Fuerza Aérea Colombiana, tuvimos que operar en un contexto geográfico específico como fue el departamento del Meta, en el cañón del río Duda y la Serranía de la Macarena, y determinar y conocer el territorio en el que se iba a operar era sumamente importante para nuestro éxito, teniendo en cuenta las limitaciones tecnológicas de la década de los noventa del siglo XX.

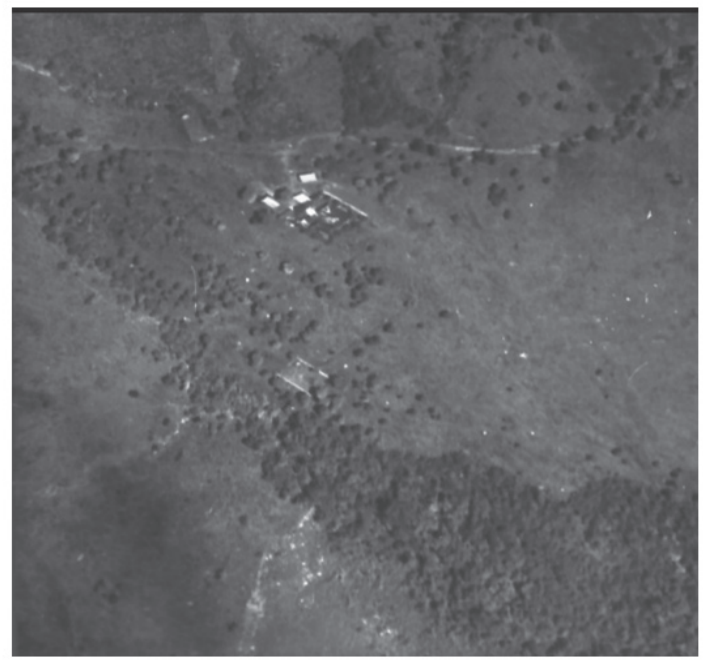

Foto 1. Campamento Casa Verde. Archivo personal

Recuperado el 11 de enero de 2018. https://dialogo-americas.com/es/articles/veinte-anos-despues-dela-operacion-colombia-contra-casa-verde 
Para las FARC, la ubicación de Casa Verde constituía un elemento estratégico de su defensa, ya que ese era el punto destinado para recibir las visitas de políticos y miembros de la sociedad civil durante el proceso de diálogo que este grupo guerrillero estableció con el gobierno de Belisario Betancur. Casa Verde estaba sumida en la vasta geografía del páramo de Sumapaz, con el fin estratégico de no ser detectados fácilmente por los helicópteros que entraban y sacaban al personal civil a sus instalaciones, lo que no representaba una amenaza para la guerrilla puesto que se encontraba a las afueras del cañón, retirada de los campamentos y era utilizada como punto de control.

Casa Verde, más que una pequeña casa en las estribaciones de la Cordillera Oriental, en el municipio de La Uribe (Meta), era un lugar simbólico para la guerrilla de las FARC. Este territorio se consolidó como su retaguardia estratégica y en centro emblemático de su lucha. Casa Verde era el nombre con que se conoció una amplia zona bajo el control de las FARC, en la región montañosa del Meta, integrada por La Gaucha, Casa Verde, la Escuela de Cuadros y El Rincón de los Viejitos.

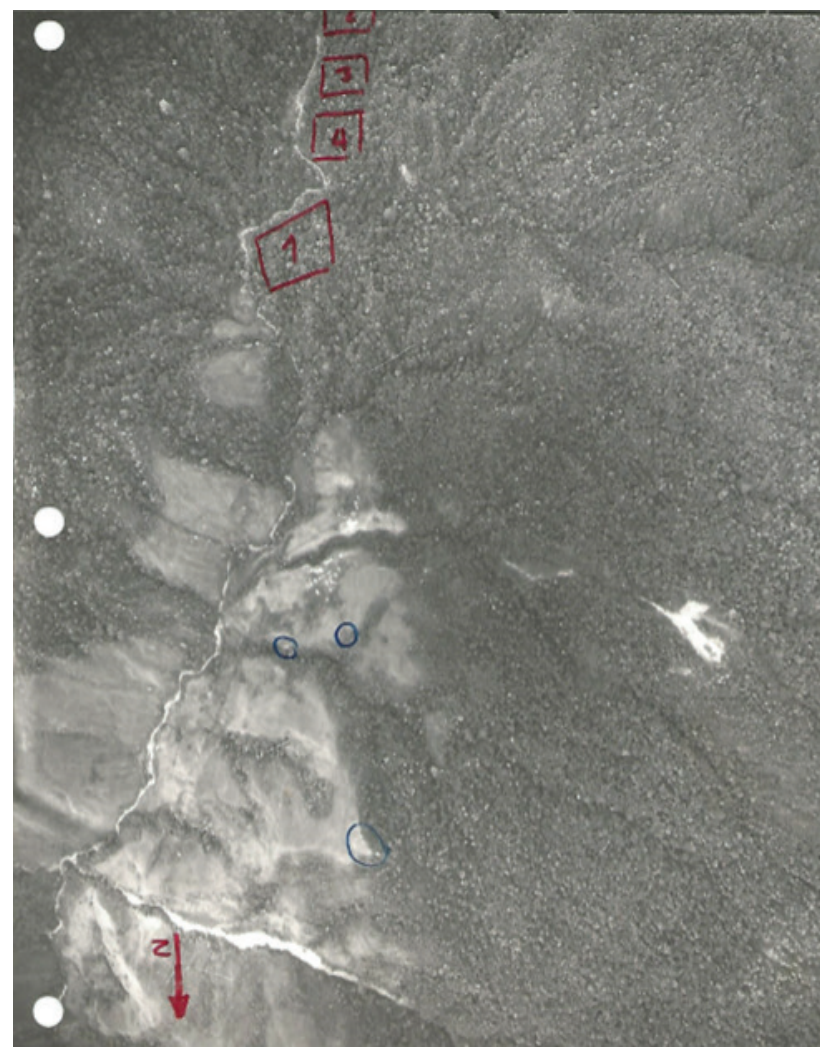

Foto 2. Campamento Casa Verde y objetivos focalizados. Archivo personal

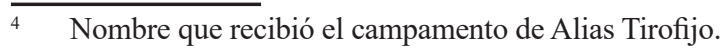


Una escuela de entrenamiento, el campamento de Tirofijo y el campamento de Reyes, entre otros, fueron los objetivos militares que se habían establecido para dar inicio a la Operación Colombia. Por los fenómenos meteorológicos y las características del territorio tales puntos eran poco visibles, pero a pesar de ello podíamos prever el lugar donde los helicópteros tenían que aterrizar, razón por la cual la orden era que primero se debía bombardear esa zona en caso de que hubiera minas antipersona. Debíamos dejar limpia esa zona para poder entrar y efectuar en su totalidad la Operación.

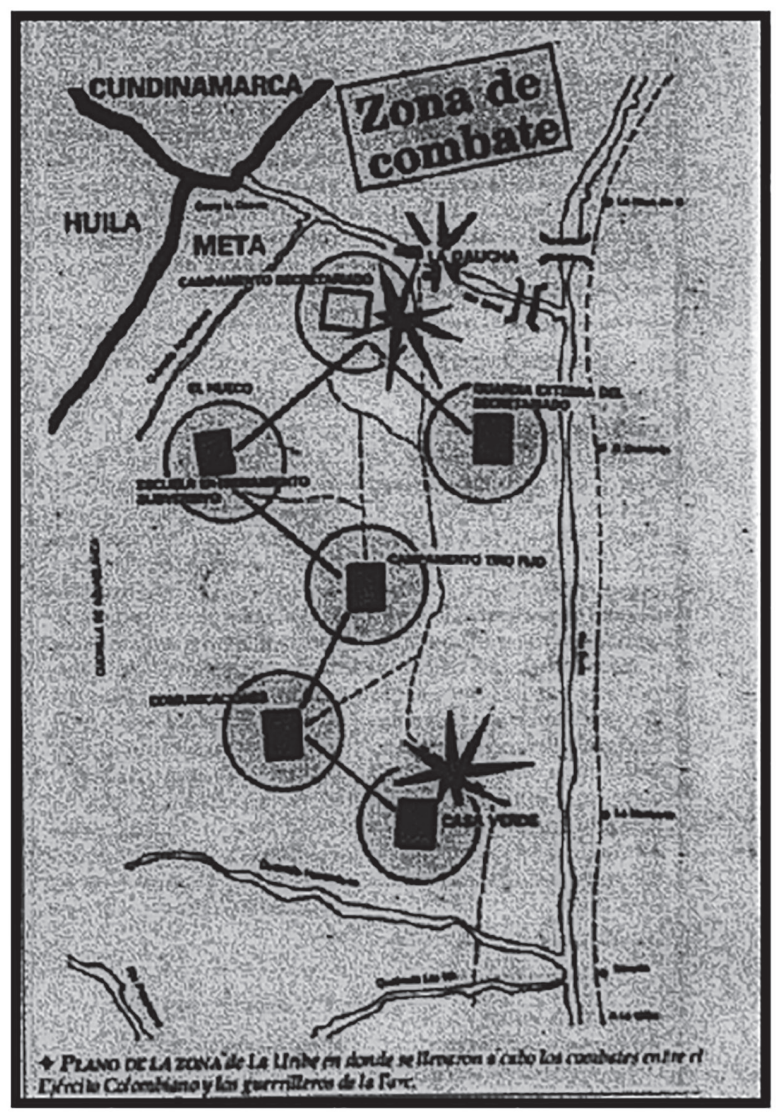

Foto 3 Mapa sobre puntos estratégicos de la Operación Colombia. Tomado de El Nuevo Siglo el 14 de diciembre de 1990.

Para las FARC, esta zona tenía una importancia simbólica ya que en ella se encontraba la tumba de Jacobo Arenas, uno de sus máximos dirigentes históricos. Es así como se entiende que el sector de Casa Verde tenía una importancia central para la guerrilla, y su control sobre el territorio permanecía incuestionado. 


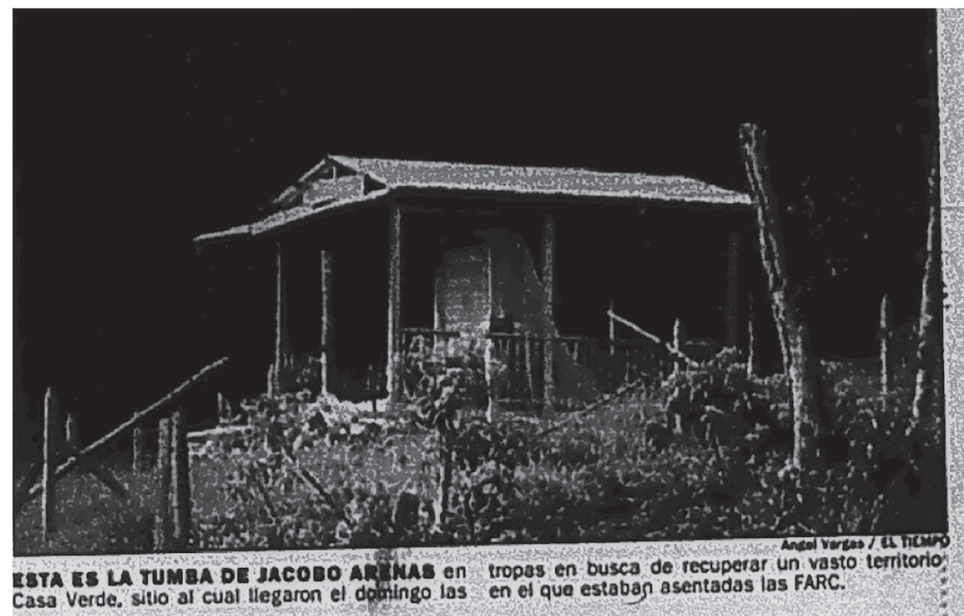

Foto 4. Tumba de Jacobo Arenas en Casa Verde. Tomado: El Tiempo, 13 de diciembre de 1990.

En ese entonces, el líder de la escuadrilla de KFIR era el general Morales, que en aquel momento era Teniente Coronel y segundo comandante de la Base; como Jefe de Operaciones del Grupo de Combate, y bajo ese cargo estuve liderando todo el planeamiento y la ejecución de la tarea. Si bien en la escuadrilla el general Morales era el líder, en la Operación Colombia tuve un liderazgo importante dentro de la base. Por eso para mí esta fue una operación de mucha importancia en mi vida profesional como oficial de la Fuerza Aérea Colombiana. Me permitió dar una conferencia en la Escuela de Guerra sobre la Operación Colombia, y guardo conmigo mucho material que fue trascendental para el éxito de esta operación.

\section{LA INTELIGENCIA MILITAR HUMANA Y EL DESARROLLO TECNOLÓGICO DE LA GUERRA}

En el caso específico de la Operación Colombia, debido a la falta de inteligencia técnica se desarrolló la inteligencia humana, para poder obtener el éxito de la Operación. El reconocimiento de los grupos guerrilleros y de los puntos de guardia y de control de la zona de Casa Verde, fue la clave para que nosotros pudiéramos reconocer el territorio y saber con claridad en dónde debíamos entregar las armas y tener el impacto que se había planeado con anterioridad. El nivel de detalle del conocimiento del territorio fue fundamental para tener todo tipo de información como las curvas de los ríos y las quebradas, y el punto de vulnerabilidad que teníamos por las cimas tan altas que componen esa geografía. En ese entonces no se hablaba de coordenadas, ni de GPS, sino del conocimiento de la inteligencia humana de haber caminado por mucho tiempo toda esa zona. 
Es importante aclarar que los generales tomamos decisiones con un margen de incertidumbre muy grande, tal y como lo señala Clausewitz. A mayor tecnología menor incertidumbre, pero la incertidumbre siempre estará presente. La inteligencia humana fue poniendo poco a poco estaciones a lo largo del territorio para que la información fuera lo menos imprecisa posible. Las fotos aéreas también fueron de gran ayuda para poder tener la ubicación precisa sobre el lugar que se buscaba impactar. En la Operación Colombia se actuó bajo la incertidumbre de saber con claridad dónde estaban las ametralladoras, porque nadie en la inteligencia había podido establecer que había nidos de ametralladoras ubicadas en ciertos puntos. La incertidumbre siempre va a existir, pero el papel de un comandante es aplicar todas las estrategias posibles para reducirla.

La inteligencia humana del territorio fue sumamente importante para que las operaciones de esa época, y la Operación Colombia específicamente, tuvieran éxito, ya que lo que ofrecían las aeronaves no era suficiente para asegurar la eficiencia de la operación. La inteligencia más la precisión fue lo que nos ayudó a tener buenos resultados; una inteligencia de última generación. El problema frente a este tema radica en que en el imaginario colectivo, cuando se habla de entrega de armas por parte de la Fuerza Aérea Colombiana, se piensa que siempre se ha tenido la misma tecnología del GPS y de la precisión con que hoy en día cuentan las aeronaves, y no se cuestiona lo difícil y riesgoso que era operar con la tecnología de hace 20 años o inclusive más. A nosotros nos tocó depender del tiempo, del rumbo y de un mapa.

Bajo este entorno, considero que se debe ahondar en lo que yo denomino la cultura de la guerra ${ }^{5}$, para hacer referencia a la manera en la que se operaba en cada uno de los contextos de la historia colombiana contemporánea. Los hechos y las operaciones ejecutadas antes de 1991 estaban regidos bajo la Constitución de 1886, en la que los parámetros del Derecho Internacional Humanitario eran distintos. Por esta razón el contexto operacional debe partir de todos los ámbitos posibles para dar cuenta de su función y de la manera estratégica del pensamiento militar que ejercía en cada momento histórico.

El caso de la Operación Colombia debe ser leído desde la tecnología y la manera de operar bajo una cultura de la guerra propia de 1990, muy diferente a la evolución y al desarrollo que ha tenido hasta hoy, para garantizar la efectividad de las operaciones y el cumplimiento de las leyes bajo el marco del DIH. Existe una concepción del DIH que el soldado actual tiene muy clara, y que hace 20 años era diferente debido a la forma como se enseñaba la doctrina militar.

Este concepto hace parte de una lista de elementos que se van a desarrollar más adelante con mayor profundidad en una publicación que saldrá en el año 2019. 


\section{DeSARROLlO TECNOLÓGICO, DISCIPLINA MILITAR Y CULTURA DE GUERRA}

La disciplina militar de los años sesenta y setenta era distinta a la que se vive hoy en día. La cultura de guerra de los ejércitos de la antigua Rusia, Yugoslavia o Alemania era muy diferente en un contexto claro de guerra internacional, de lo que puede ser en la actualidad. Es decir, si la Operación Colombia se hubiera ejecutado con la tecnología actual, habría sido mucho más eficiente, evitando errores que en aquel momento era imposible no cometer.

A pesar de que el desarrollo tecnológico iba de la mano tanto de la Fuerza Pública como de los grupos armados ilegales, fue solo hasta los primeros años del siglo XXI cuando la Fuerza Aérea tomó la delantera al adoptar una mejor tecnología y condiciones más favorables para posicionarse de una forma ventajosa sobre los grupos guerrilleros y de autodefensa ilegal. Se registró entonces la adopción de importantes avances tecnológicos por parte de la Fuerza Aérea Colombiana; aunque a la vez la contraparte consiguió en el mercado negro armas y municiones y atacó intensamente a la Fuerza Pública y a la población civil ${ }^{6}$.

Sumado a todo lo anterior, cabe señalar que para la cultura militar de la época era normal, e inclusive representaba un honor, darles a los medios de comunicación explicaciones operativas cuando se tenía éxito en alguna operación. Aspecto negativo, sin embargo, en tanto esa información era aprovechada por los enemigos para entender las dinámicas y estrategias que estaba utilizando la Fuerza Aérea Colombiana.

Es importante entender que el Plan Colombia y las acciones del Estado fueron claves para que crear cultura militar y desarrollo estratégico. De ahí que dicho progreso estuviera por encima del enemigo y hubiera sido posible llegar al Plan Victoria, que hoy en día enorgullece a toda la Fuerza Pública y en especial a la Fuerza Aérea, por disponer de un poder aéreo que determina un desequilibrio en un campo de batalla en el cual las aeronaves de la FAC tuvieron y cumplieron con lo necesario para garantizar y ejercer la soberanía. La Operación Colombia fue entonces una operación que dio cuenta de la adaptación constante de la Fuerza Pública a cada contexto y de la capacidad del poder aéreo empleado por la Institución en ese momento.

Antes de 2006 nosotros operábamos con unas aeronaves con las que se combatía mucho, como los T27, A37, Mirage y el KFIR. No obstante, con este tipo de aviones teníamos que volar con un mapa en la mano para poder ubicar

Un ejemplo de ello son las tomas y ataques guerrilleros a las cabeceras municipales durante la década de los años noventa; fenómeno histórico de gran barbarie y atrocidad, que exigió una respuesta integral por parte de la Fuerza Pública. En particular la Fuerza Aérea Colombiana desarrolló un mecanismo de diagnóstico, monitoreo y acción que fue muy efectivo en contrarrestar dicha amenaza. Este esfuerzo institucional será documentado en un libro que publicaré en el año 2019. 
el blanco militar. Aun cuando los dos últimos tenían un sistema inercial de navegación, este no era tan preciso como los sistemas de última generación. Afortunadamente, todo eso ha venido cambiado, ya que nuestra precisión no se podía calcular inequívocamente y teníamos que lidiar con los recursos e infraestructura de ese entonces. En 2006 llegan a Colombia los aviones Súper Tucano y empiezan a reemplazar a muchas de las aeronaves antiguas para este tipo de operaciones. Esto nos permitió atacar a 20.000 pies sin ser detectados, ni tener el riesgo de que nos pudieran impactar desde la tierra. No se puede decir que el nivel de tecnología con el que se contaba en ese entonces se debía a negligencia del Estado para invertir en el gasto militar, sino que tal era la tecnología con la que se contaba en el mundo militar de la época.

En la medida en que fue llegando mejor tecnología, mejores fueron los resultados. La demora de poder poner la coordenada correcta en una operación con despliegue de armamento en el sistema de cada avión, se debía al trabajo de insertar la coordenada en el sistema, porque la bomba era guiada por GPS. Solo hasta el año 2006 pudimos contar con un tipo de tecnologías de precisión con aviones como el Súper Tucano, que en términos prácticos hace lo mismo que el KFIR.

Ya en 2010 se contaba con los KFIR C10 con el sistema de navegación con un GPS que genera información mucho más precisa. La precisión pasó de ser de millas a metros. Desde la época de 1990 hasta 2008 se utilizaba el sistema inercial en los KFIR, que determinaba la posición geográfica a medida que iba avanzando la aeronave, razón por la cual la operatividad de la época en la que se ejecutó la Operación Colombia, no era tan precisa como la que se obtiene con los aviones de hoy en día.

\section{A MOdo DE CONCluSión: TransformaCiOnes EN EL CONTEXTO OPERACIONAL}

Así como en el contexto de la Operación Colombia la constante era la incertidumbre y la imposibilidad de garantizar un control absoluto del devenir operacional, las décadas del noventa y del 2000 van a estar caracterizadas por el desarrollo del proceso de inteligencia militar, de la tecnología (en el caso de la Fuerza Aérea Colombiana va a darse una inversión y adecuación de las aeronaves fundamental para confrontar a los grupos armados ilegales), del entrenamiento militar contraguerrillero y de la voluntad política de los gobiernos centrales, que incidieron directamente en la capacidad de transformar las dinámicas del conflicto, generando éxitos militares y cambios en las dinámicas de seguridad.

Todas estas transformaciones en el terreno del contexto operacional incidieron de manera positiva en el fortalecimiento de una cultura militar, ahondando en la disciplina y la mística, y elevando la moral al interior de las 
instituciones castrenses. Este cambio es fundamental, ya que potenció el capital humano, desarrolló las posibilidades y capacidades del personal adscrito a las Fuerzas Militares, convenciéndoles de que la victoria era factible y accesible, y fortaleciendo las relaciones con la sociedad y con el poder político, elementos claves para garantizar y potenciar la legitimidad histórica de su actuar.

La forma de operar de las Fuerzas Militares, y en particular de la Fuerza Aérea Colombiana, van a evidenciar su capacidad de incidir en las dinámicas del conflicto de manera plena en contextos operacionales como los de la Zona de Distensión o en la Operación Vuelo del Ángel, contextos en los que se puso de presente que la nueva estructura organizacional iba a garantizar la victoria para el Estado colombiano, teniendo una fuerte influencia las capacidades operacionales del poder aéreo.

Este proceso de racionalización del uso del poder aéreo quedó plasmado formalmente en el documento Manual de Estado Mayor Fuerza Aérea, en el que se evidencia que el proceso de cualificación no fue solamente en la parte tecnológica, sino en la planeación y estrategia interna: "El Macro proceso de Planeación, Programación y Presupuestación de la Fuerza Aérea Colombiana (PPBS), se lidera y desarrolla a través del Sistema Integrado de Planeación que corresponde a la integración y articulación de los Estados Mayores de Planeamiento y Coordinación para los diferentes niveles de planeamiento de la Fuerza" (2003, p. 11).

Otro aspecto esencial en el fortalecimiento de la Fuerza Aérea fue el de implementar el principio del poder aéreo de "control centralizado y ejecución descentralizada" mediante la organización del Sistema de Comando y Control de la Fuerza Aérea. Este sistema integró todas las actividades de inteligencia, planeación, ejecución y control operacional, y además todos los elementos tecnológicos de comunicaciones, tecnologías de la información, señales de radar, sensores y plataformas de inteligencia, bajo el Centro de Comando y Control de la Fuerza Aérea y sus organizaciones subalternas en los Comandos y Grupos Aéreos, los Centros de Comando y Control de las Bases Aéreas. Esto le permitió al Comandante de la FAC tener un control de todas las operaciones, explotar adecuadamente la flexibilidad, velocidad, precisión y versatilidad del poder aéreo e incorporar el DIH en toda la estructura operacional, mediante la definición de las reglas de encuentro, los informes de misión cumplida, el control cerrado de las operaciones de interdicción del narcotráfico y el sistema de información operacional.

En este proceso de desarrollo de las capacidades institucionales, cumple un papel fundamental -más no exclusivo- el Plan Colombia, en la medida en que permite que todas las variables enunciadas al inicio de este documento se potencien y desarrollen, de forma sistemática para lograr la victoria sobre los grupos armados ilegales que tenían amenazada a la sociedad colombiana y a la infraestructura del Estado. 
Esta afirmación no es particular del autor de este trabajo; se encuentra transversalizada en la mentalidad de los miembros de las Fuerzas Militares, tal y como lo reconoce el siguiente testimonio: "La Fuerza Aérea es una antes del Plan Colombia, y otra después del mismo; ganamos en capacidad militar y operativa; fortalecimos la inteligencia propia y conjunta; recibimos asesoría especializada que nos permitió comprender el mejor uso que podíamos hacer de nuestros recursos... Cuando nos pidieron retomar la Zona de Distensión teníamos la capacidad real de hacerlo, atacando ordenada, sistemática y simultáneamente en varios puntos estratégicos... Las FARC no esperaban esa capacidad de respuesta, por eso los tomamos por sorpresa y les golpeamos en esa medida" (MARÍn et al. 2016, p. 238).

Este proceso de transformación y modernización permitió que la Fuerza Aérea Colombiana desarrollara exitosas operaciones aéreas en contra de los grupos armados ilegales, llevando a la desmovilización de las estructuras armadas de las AUC y de las FARC, y al debilitamiento estratégico de otras organizaciones.

Entre 1999 y 2006 se presentan las operaciones aéreas más importantes y significativas en la historia del país, desarrollando una estrategia ofensiva que va a garantizar una mayor capacidad de control efectivo del territorio, y que va a impedir que grupos ilegales consoliden una "guerra de movimientos", con la cual se habrían podido apoderar de vastas zonas del territorio, afectando la calidad de vida de la población que allí habitaba. Al contrario, como se ha mostrado con los trabajos de Echandía (2013) y SALAS (2014), las dinámicas de seguridad del país cambian significativamente después del año 2006.

Pero no solo hay un cambio en el contexto social; también hay una transformación poderosa al interior de las Fuerzas Militares, proceso que no ha sido abordado por la academia, y que es fundamental para comprender la profesionalización, modernización y tecnificación que estas instituciones desarrollaron, y que permitieron que alcanzaran la victoria.

\section{REFERENCIAS}

Alvarado, Y. P. (2006). Operación Vuelo de Ángel la retoma de Mitú. En Osorio Martínez, G. (Comp.), Hablan los generales las grandes batallas del conflicto colombiano contadas por sus protagonistas. Bogotá: Norma.

Comisión Nacional de Reparación y Reconciliación -CNRR (2009). Recordar y narrar el conflicto. Herramientas para reconstruir memoria histórica. Bogotá: CNRR.

DÁvila, A. et al. (2016). El conflicto en contexto. Análisis de 5 regiones. Bogotá: Pontificia Universidad Javeriana. 
ECHANDÍA, Camilo (2013). Punto de partida del debilitamiento de las FARC. En Revista Zero, Bogotá: Universidad Externado de Colombia.

Fundación Ideas para la Paz (2013). Hoy y ayer del Bloque Oriental de las FARC. Bogotá: FIP.

MARÍn et al. (2016). Victorias desde el aire: La Fuerza Aérea Colombiana y el término del conflicto armado. Bogotá: Editorial Ibáñez.

RuEdA, R. P. (2004). La transformación de la Guerra. En La Historia de las Guerras. Bogotá: Ediciones B.

SALAS, Luis Gabriel. (2014) Lógicas territoriales y relaciones de poder en el espacio de los actores armados: un aporte desde la geografía política al estudio de la violencia y el conflicto armado en Colombia, 1990-2012 En Cuadernos de Geografía, Bogotá, Universidad Nacional de Colombia.

SÁnchez SuÁrez, Pedro Arnulfo. (CR). (2014). Poder aéreo en la seguridad y defensa nacional. En Revista Taktika $\mathrm{N}^{\circ}$ 5. Colombia, Fuerza Aérea Colombiana.

SAnTos, María Alejandra. (2014). El poder de la Fuerza Aérea Colombiana: la Operación Vuelo de Ángel y el cambio en la asimetría de poder entre las FF.MM y las FARC-EP. Bogotá, Universidad Colegio Mayor de Nuestra Señora del Rosario, Facultad de Ciencia Política y Gobierno. 